\title{
Prevalencia y Características Morfométricas del Foramen Venoso en Cráneos del Noreste de México
}

\author{
Prevalence and Morphometric Characteristics Foramen Venosum in Northeastern Mexico Skulls
}

\author{
J. C. Aviles-Solis; A. Olvera-Barrios; O. De la Garza Castro; R. E. Elizondo-Omaña \& S. Guzmán-López
}

AVILES-SOLIS, J. C.; OLVERA-BARRIOS, A.; DE LA GARZA, C. O.; ELIZONDO-OMAÑA, R. E. \& GUZMÁN-LÓPEZ, S. Prevalencia y características morfométricas del foramen venoso en cráneos del Noreste de México.Int. J. Morphol., 29(1):158-163, 2011.

RESUMEN: El foramen venoso es una estructura inconstante que se encuentra posterior al foramen redondo y medial al foramen oval, y da paso a una vena que anastomosa al plexo venoso pterigoideo con el seno cavernoso. La existencia de éste foramen puede ser motivo de complicaciones clínicas, entre ellas que es una potencial vía de acceso al seno cavernoso de trombos infectados y constituye una posible falsa vía durante la rizotomía percutánea del trigémino pudiendo puncionar la vena y ocasionar hematomas subdurales. Se utilizó la colección de cráneos del Departamento de Anatomía de la Facultad de Medicina UANL. Se observó la frecuencia y se midieron los siguientes parámetros del foramen: Ancho y largo; en la cara interna de la base del cráneo se midieron las distancias a: foramen redondo, foramen oval, plano sagital mediano y entre forámenes venosos; en la cara externa de la base del cráneo se midieron las distancias con: foramen oval, plano sagital medio y entre los forámenes venosos. Las medidas fueron llevadas a cabo por tres observadores distintos a través de medición directa con vernier y con microfotografías con escalas milimétricas. Se encontró una frecuencia del $20 \%$ con medidas medias de 1,66mm de largo, 1,06mm de ancho; en la cara interna de la base del cráneo: 11,31mm de distancia al foramen redondo, 4,13mm al foramen oval, $17,75 \mathrm{~mm}$ al plano sagital medio y $31,91 \mathrm{~mm}$ entre los forámenes; en la cara externa de la base: $2,50 \mathrm{~mm}$ al foramen oval, $19,54 \mathrm{~mm}$ al plano sagital medio y $36,05 \mathrm{~mm}$ entre forámenes venoso. La distancia al foramen oval varía en la cara interna y la cara externa de la base del cráneo debido a un trayecto oblicuo del foramen que tiende a aproximarse al foramen oval y por tanto a separarse del plano sagital medio. Los forámenes derechos fueron generalmente más grandes.

PALABRAS CLAVE: Hueso esfenoides; Base de cráneo; Fosa craneal media; Foramen venoso.

\section{INTRODUCCIÓN}

Estructuralmente el cráneo está conformado por 22 huesos, que clásicamente se dividen en 14 que conforman el viscerocráneo y 8 que constituyen el neurocráneo. El esfenoides forma parte de la base del cráneo y se encuentra en el centro de ésta articulándose a su vez con todos los huesos del neurocráneo. Debido a ésta disposición los antiguos anatomistas le dieron su nombre a partir de la raíz griega sphenon que significa cuña (Testut, 1924).

En el borde interno del ala mayor del esfenoides se encuentra una semiluna de forámenes de concavidad anterolateral de los cuales 4 son constantes (fisura orbitaria superior, foramen redondo, oval y espinoso) y 2 inconstantes (foramen venoso y foramen petroso). El foramen venoso (de Vesalio) se encuentra posterior al foramen redondo y medial al foramen oval, y da paso a una vena emisaria que anastomosa al plexo venoso pterigoideo con el seno cavernoso (Testut). La frecuencia con que se ha encontrado es muy variable con cifras que van desde el 17\% (Reymond et al., 2005) hasta el 80\% (Gingsberg et al., 1994). Partiendo de la premisa que cualquier foramen del cuerpo es resultado del encuentro de dos centros de osificación distintos, el foramen venoso marca el sitio donde la osificación endocondral (correspondiente a la base, condrocráneo) se encuentra con la osificación membranosa (desmocráneo) (James et al., 1980).

La existencia de éste foramen puede ser motivo de complicaciones clínicas. Al dar paso a una vena emisaria, es una potencial vía de comunicación entre el plexo venoso 
pterigoideo y el seno cavernoso, estructura que trombos infectados pueden utilizar para ingresar a la cavidad craneal. Se han reportado casos de infecciones dentales o faciales que se complican con la aparición de una trombosis séptica del seno cavernoso. Se cree que la principal vía de acceso de los trombos infectados es a través del plexo venoso pterigoideo (Embong et al., 2007; Jiménez et al., 2004; Ebright et al., 2001).

Durante la rizotomía percutánea del nervio trigémino mediante la inyección de glicerol, por radiofrecuencia y descompresión con balón, procedimientos en los que se introduce una aguja hasta el foramen oval, la existencia del foramen venoso puede causar confusión al introducir accidentalmente la aguja en éste último y perforar la vena emisaria que contiene, causando complicaciones graves, dentro de las que podemos citar a los hematomas temporales (Håkanson, 1981; Martínez et al., 2007). Estos procedimientos son ampliamente usados en la actualidad en el tratamiento de neuralgia del trigémino (Sindou \& Tatli, 2009a; 2209b).

La asimetría del foramen venoso ha sido relacionada con la existencia de melanoma nasofaríngeo, angiofibroma y fístulas carótico - cavernosas con drenaje a través de la vena emisaria (Lanzieri et al., 1988). En la literatura encontrada existe una diferencia de opiniones en cuanto a estos casos ya que otro estudio realizado por Gingsberg et al. no se encontró relación entre la asimetría del foramen y patología alguna.

Determinar la frecuencia, características y simetría del foramen venoso es de importancia para que los profesionales que realicen procedimientos, como rizotomías percutáneas en el área tengan presentes las posibles variaciones de la misma. Es necesario conocer la existencia y características del mismo cuando se revisan tomografías de la base del cráneo para evitar confundir variaciones normales con hallazgos anormales (Keskil et al., 2003).

Existen pocos estudios en la literatura que describen las características de este foramen y variaciones en la descripción del mismo. Debido a la poca literatura y a la variación de los resultados, este estudio analizó la frecuencia y características. No existen estudios realizados en población mexicana. Es necesario recabar datos y reportes sobre esta estructura anatómica.

\section{MATERIAL Y MÉTODO}

Este es un estudio observacional, transversal, no comparativo (descriptivo). Se utilizó la colección de crá- neos existente en la osteoteca del Departamento de Anatomía de la Facultad de Medicina UANL. Se excluyeron aquellos cráneos que se encontraron en mal estado o que presentaban rupturas en el área de estudio (Ala mayor del esfenoides). Se observó el área comprendida entre el foramen oval y el foramen redondo en busca de otros forámenes. Se utilizó una fibra de Nylon de 0,1 $\mathrm{mm}$ de grosor y $10 \mathrm{~mm}$ de longitud para comprobar la permeabilidad del foramen. Se tomaron medidas de largo y ancho del foramen, así como las distancias existentes desde el borde del foramen hasta el borde del foramen redondo (Fig. 1a) y oval (Fig. 1b) en la cara interna de la base del cráneo. En la cara externa de la base del cráneo se midió solamente la distancia al foramen oval. Se consideró como largo al diámetro mayor y ancho al diámetro menor. En el caso de encontrarse bilateralmente se midió la distancia entre los forámenes venoso tanto en la cara interna como en la cara externa del cráneo. Se midió también la distancia existente desde el borde del foramen hasta el plano mediano tomando como punto de referencia la prolonga-

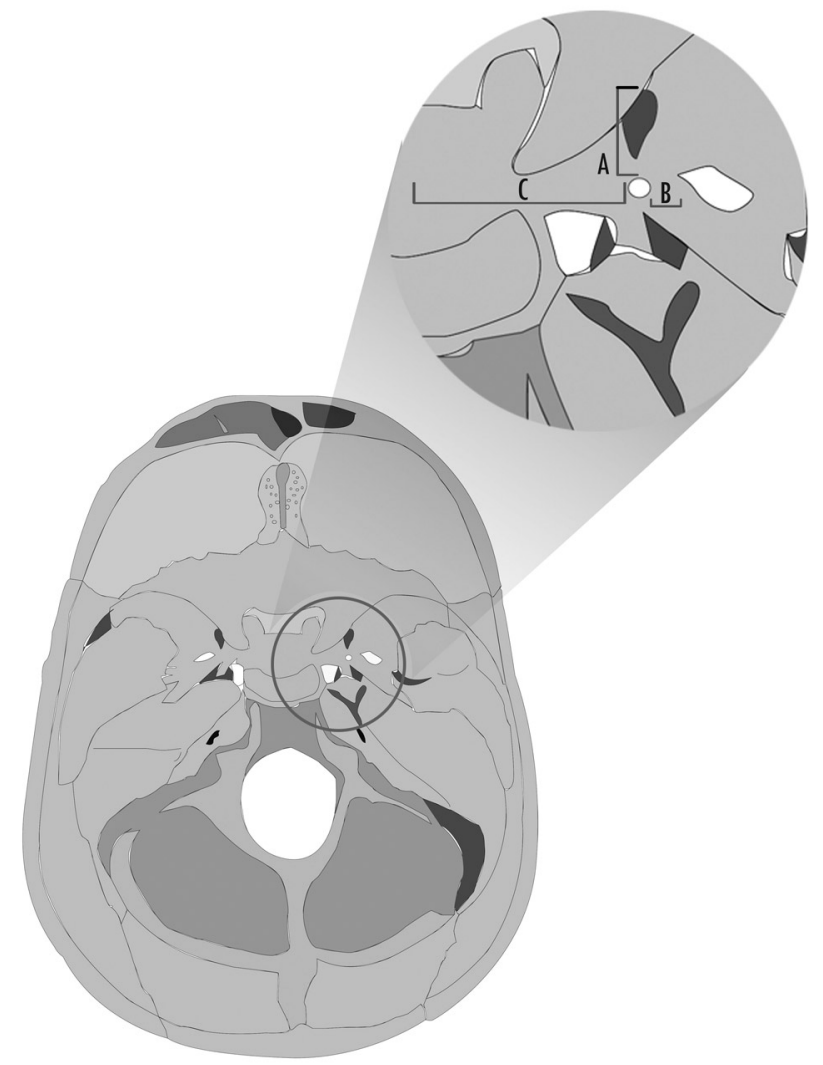

Fig. 1. Esquema de la base de cráneo con acercamiento a la fosa craneal media. A. Distancia entre foramen venoso y foramen redondo. B. Distancia entre el foramen venoso y foramen oval. C. Distancia entre foramen venoso y el plano mediano. 
ción del surco del seno sagital superior, proceso crista galli y lámina perpendicular del etmoides (Fig. 1c). La medición fue realizada por tres observadores independientes y cegados entre sí a través de dos métodos, el primero por medio de fotografías con escalas milimétricas al margen obtenidas mediante una cámara adosada a un microscopio binocular Carl Zeiss con un objetivo panorámico 4x y 10x midiéndose las distancias sobre la imagen con una regla y realizando las conversiones necesarias de acuerdo a la escala, el segundo método fue a través de la medición directa con un vernier electrónico con una resolución de 0,01 $\mathrm{mm} / .0005$ " y un error máximo de $20 \mu \mathrm{m}$ en $100 \mathrm{~mm}$. Se obtuvieron seis lecturas para cada parámetro. Se tomó la media de las medidas en vernier y se sumó con la media fotográfica para obtener una media final. Los datos obtenidos fueron procesados con estadística descriptiva y reportados en Tablas.

\section{RESULTADOS}

Se encontró la presencia del foramen venoso (Fig. 2) en 5 cráneos distintos $(n=25)$ con un total de 6 forámenes (Tabla I). En dos ocasiones se encontraron indicios de lo que pudiera ser un foramen pero no fueron considerados como positivos por no ser permeables a la fibra. En algunos cráneos pudo observarse un surco corriendo paralelo a la unión del ala mayor del esfenoides con el cuerpo del mismo con un foramen en su centro. Las medias finales se reportan en la Tabla II.

Tabla I. Cantidad de forámenes totales encontrados en la muestra y su distribución por lados del cráneo donde fueron observados.

\begin{tabular}{lcc}
\hline Forámenes (n) & Lado Derecho & Lado izquierdo \\
\hline 6 & 3 & 3 \\
$100 \%$ & $50 \%$ & $50 \%$ \\
\hline
\end{tabular}

Fig. 2. Fosa Craneal media. Acercamiento al ala mayor del esfenoides izquierda de una muestra respresentativa de los cráneos estudiados, en donde se aprecia claramente el Foramen venoso. Fotografía (izquierda) y esquema (derecha).
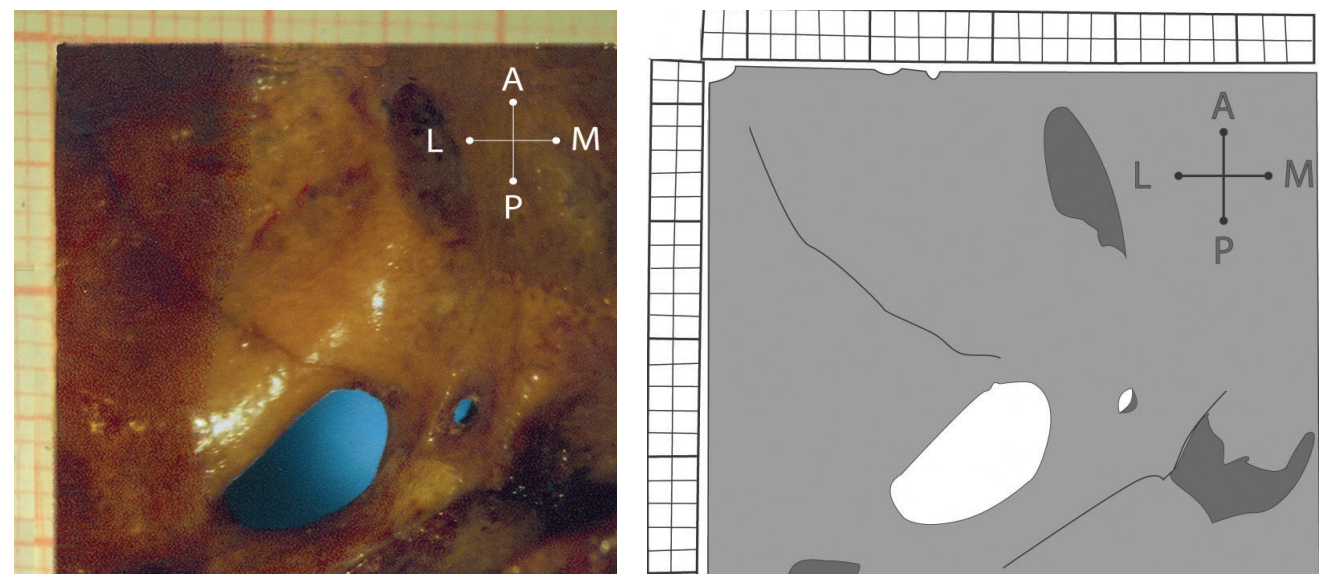

Tabla II. Medidas con desviaciones estándar con los datos obtenidos con el Vernier y las micrografías para cada parámetro agrupadas por lado y media final. Medidas expresadas en $\mathrm{mm}$.

\begin{tabular}{lccc}
\hline Foramen Venosum & $\begin{array}{c}\text { Lado izquierdo } \\
\text { Media (mm), SD }\end{array}$ & $\begin{array}{c}\text { Lado derecho } \\
\text { Media (mm), SD }\end{array}$ & $\begin{array}{c}\text { Media Final } \\
\text { Media (mm), SD }\end{array}$ \\
\hline Largo & $1,25 \pm 0,25$ & $2,07 \pm 0.38$ & $1,66 \pm 0,53$ \\
Ancho & $0,78 \pm 0,15$ & $1,34 \pm 0.42$ & $1,06 \pm 0,42$ \\
Vista superior & $12,06 \pm 3,28$ & $10,55 \pm 1.39$ & $11,31 \pm 2,40$ \\
Distancia al foramen redondo & $4,24 \pm 0,81$ & $4,03 \pm 1.17$ & $4,13 \pm 0,91$ \\
Distancia al foramen oval & $17,95 \pm 2,63$ & $17,55 \pm 3.98$ & $17,75 \pm 3,03$ \\
Distancia al plano mediano & ---- & ---- & 31,91 \\
Distancia entre forámenes & & $2,51 \pm 0.28$ & $2,49 \pm 0.35$ \\
Vista inferior & $2,46 \pm 0,48$ & $19,18 \pm 1.38$ & $19,54 \pm 1.99$ \\
Distancia foramen oval & $19,91 \pm 2,76$ & --- & 36,05 \\
Distancia plano mediano & ---- & & \\
Distancia entre forámenes & & \\
\hline
\end{tabular}




\section{DISCUSIÓN}

La frecuencia del foramen venoso en el presente estudio fue de $20 \%(\mathrm{n}=5)$. Si bien en otros 2 cráneos se encontraron indicios de lo que pudiera ser el foramen estos fueron descartados debido a que no tenían una estructura equivalente en la cara externa de la base del cráneo y no eran permeables a la fibra. A pesar de que en el estudio realizado por Kodama et al. (1997), donde el foramen venoso fue clasificado como de tipo abierto y de tipo cerrado, sólo se han tomado en cuenta los forámenes completamente permeables a la fibra. Un estudio en Polonia reporta una frecuencia de 17\% (Reymond et al.), mientras que en un estudio en Japón se encontró en 55\% de los cráneos juveniles y en $21 \%$ de los cráneos adultos (Kodama et $a l$.$) , en tanto que un estudio americano el foramen existía en$ $80 \%$ del total de su muestra (Gingsberg et al.) y un estudio en Nepal obtuvo una frecuencia de $42 \%$ (Gupta et al.).

Se encontró de manera bilateral solamente en uno de los 5 cráneos. La incidencia del lado izquierdo $(n=3)$ fue igual que en el lado derecho $(n=3)$. En otros estudios se ha reportado una incidencia principalmente bilateral del foramen (Kodama et al.; Gupta et al.). En este estudio la frecuencia de la bilateralidad de los forámenes en el total de la muestra fue de 1 en 25 cráneos (4\%).

El tamaño del foramen varía también con la edad, aumenta su anchura, largo y su distancia al plano sagital medio. El crecimiento ocurre principalmente antes de los 5 años (Sgouros et al.). En la Tabla III se observa la comparación de medidas entre las distintas edades y con el estudio actual.

La distancia al foramen oval cambia entre la cara interna y cara externa de la base del cráneo, por lo que se concluye que los forámenes no tienen un trayecto vertical sino oblicuo tendiendo a separarse del plano sagital medio y aproximándose al foramen oval. En los otros estudios revisados no se indica un trayecto oblicuo del foramen. Encontramos en general que los forámenes del lado derecho son más largos y anchos que los del lado izquierdo. En los parámetros relativos a la distancia con el plano sagital medio solo fue tomada la media de las medidas con el vernier debido a que en algunas fotografías fue difícil ubicar el punto medio con exactitud.
No pudimos lograr una comparación de la presencia del foramen y sus características entre hombres y mujeres debido a la falta de información de los cráneos existentes en la osteoteca del Departamento de Anatomía. Se ha mencionado en estudios anteriores que no existe diferencia significativa entre ambos (Kodama et al.).

La relación entre la normalidad o anormalidad de la asimetría del foramen no pudo ser evaluada debido a que sólo un cráneo presentaba el foramen de manera bilateral siendo insuficiente el número para poder establecer una relación de normalidad o anormalidad en las medidas del foramen.

El foramen venoso es una estructura inconstante que a pesar de que su frecuencia no es muy elevada en la población, el ignorar su posible existencia puede generar complicaciones en procedimientos como la rizotomía del trigémino por medio de la inyección de glicerol, o en la posible propagación de infecciones del macizo facial cuyo drenaje venoso involucre el plexo pterigoideo. Es necesario también conocer su prevalencia para evitar confundir hallazgos radiológicos normales con posibles lesiones.

Las características descritas pueden ser de utilidad para tener un referente en cuanto a sus medidas y ubicación que pudieran ser relevantes para reducir la tasa de falsas rutas y complicaciones potencialmente graves durante las rizotomías percutáneas y bloqueos del ganglio trigeminal, futuros estudios y procedimientos experimentales. Estas características cambian ligeramente con la edad. También concluimos que los forámenes tienen trayecto oblicuo que tiende a separarse del plano sagital medio y se aproxima al foramen oval en la cara externa de la base del cráneo en comparación con la cara interna de la base.

Debido al tamaño de la muestra y la frecuencia del foramen, así como la falta de datos sobre la presencia o no de condiciones patológicas en los cráneos relacionadas con la posible asimetría del foramen, no pudimos determinar si hay alguna relación entre las patologías y la alteración de las características simétricas del foramen. Se requieren más estudios con una orientación clínica y con otros métodos adecuados para poder ser estudiado en seres vivos, tales como TAC y MRI.

Tabla III. Comparación de las medidas obtenidas en recién nacidos y adultos para comprobar el crecimiento del foramen a través de los años. También se comparan las medidas de la población adulta con las medidas obtenidas en este estudio. Medidas expresadas en mm

\begin{tabular}{|c|c|c|c|c|c|c|c|}
\hline \multirow[t]{2}{*}{ Estudio } & \multirow[t]{2}{*}{ Población } & \multicolumn{2}{|c|}{ Ancho } & \multicolumn{2}{|c|}{ Largo } & \multicolumn{2}{|c|}{ Distancia plano mediano } \\
\hline & & Derecho & Izquierdo & Derecho & Izquierdo & Derecho & Izquierdo \\
\hline Alemania (Lang et al., & Recién nacidos & 1,0 & 1,0 & 1,0 & 1,0 & 10,0 & 10,75 \\
\hline 1984) & Adultos & 1,14 & 1,3 & 2,0 & 1,4 & 15,78 & 17,62 \\
\hline Estudio actual & Adultos & 1,34 & 0,78 & 2,07 & 1,25 & 17,55 & 17,95 \\
\hline
\end{tabular}


AVILES-SOLIS, J. C.; OLVERA-BARRIOS, A.; DE LA GARZA, C. O.; ELIZONDO-OMAÑA, R. E. \& GUZMÁN-LÓPEZ, S. Prevalence and morphometric characteristics foramen venosum in Northeastern Mexico skulls. Int. J. Morphol., 29(1):158-163, 2011.

SUMMARY: Foramen venosum (foramen Vesalius) is an inconstant structure which is located posterior to the foramen rotundum and medial to the foramen ovale, and it gives pace to an emissary vein that communicates the plexus pterigoideus and the sinus cavernosus. The existence of this anatomical structure can be of interest in certain procedures like percutaneous trigeminal rhizotomy where this foramen can be the cause of a false pathway and be punctured causing a subdural hematoma. It also can be an access to the sinus cavernosus for infected thrombus coming from dental and facial infections. For this study the skull collection of the Anatomy Department, Faculty of Medicine UANL, was used. We observed the frequency and measured the following parameters: Length and width; in the basis cranii we measured: Distances to the foramen rotundum, foramen ovale, planum medianum and between two foramina venosum (one on each side). In basis cranii externa we measured: distances to foramen ovale, planum medianum, and between two foramina venosum. Three independent observers blinded among them, measured the parameters using a Vernier and microphotographs with milimetrical scales. A $20 \%$ frequency was found and the following median measures: Length $1.66 \mathrm{~mm}$, width $1.06 \mathrm{~mm}$; basis cranii: distance to foramen rotundum $11.31 \mathrm{~mm}$, distance to foramen ovale $4.13 \mathrm{~mm}$, distance to planum medianum $17.75 \mathrm{~mm}$ and $31.91 \mathrm{~mm}$ between both foramina venosum. Basis cranii externa: distance to foramen ovale $2.49 \mathrm{~mm}$, distance to planum medianum $19.54 \mathrm{~mm}$ and distance between foramenina venosum 36.05. The distance to the foramen ovale varies between basis cranii and basis cranii externa because the foramen has an oblique trajectory and it approximates to the foramen ovale (from superior to inferior) and separates from the planum medianum. It was found bilaterally only in one skull. There are differences between the left and right foramens, the latter being generally larger.

KEY WORDS: Sphenoid bone; Skull base; Middle cranial fossa; Foramen venosum.

\section{REFERENCIAS BIBLIOGRÁFICAS}

Ebright, J. R.; Pace, M. T. \& Niazi A. F. Septic thrombosis of the cavernous sinuses. Arch. Intern. Med., 161:26716,2001 .

Embong, Z.; Ismail, S.; Thanaraj, A. \& Hussein, A. Dental infection presenting with ipsilateral parapharyngeal abscess and contralateral orbital cellulitis - A case report. Malaysian Journal of Medical Sciences, 14(2):62-6, 2007.

Gingsberg, L. E.; Pruett, S. W.; Chen, M. Y. \& Elster, A. D. Skull base foramina of the middle cranial fossa: reassessment of normal variation with high resolution CT. Am. J. Neuroradiol., 15:283-91, 1994.

Gupta, N.; Ray, B. \& Ghosh, S. Anatomic characteristics of foramen Vesalius. Kathmandu Univ. Medic. J., 3(2):1558,2005 .

Håkanson, S. Trigeminal neuralgia treated by the injection of glycerol into the trigeminal cistern. Neurosurgery, 9: 638-46, 1981.

James, T. M.; Presley, R. \& Steel, F. L. The foramen ovale and sphenoidal angle in man. Anat. Embryol., 160(1):93104, 1980.

Jiménez, Y.; Bagán, J. V.; Murillo, J. \& Poveda, R. Infeccio- nes odontogénicas. Complicaciones. Manifestaciones sistémicas. Med. Oral Patol. Oral Cir. Bucal, 9 Suppl:S143-7:39-43, 2004.

Keskil, S.; Gözil, R. \& Calgüner, E. Common surgical pitfalls in the skull. Surg. Neurol., 59(3):228-31, 2003.

Kodama, K.; Inoue, K.; Nagashima, M.; Matsumura, G.; Watanabe, S. \& Kodama, G. Studies on the foramen Vesalius in the Japanese juvenile and adult skulls. Hokkaido Igaku Zasshi, 72:667-74, 1997.

Lanzieri, C. F.; Ducchesneau, P. M.; Rosenbloom, S. A.; Smith, A. S. \& Rosenbaum, A. E The significance of asymmetry of foramen of Vesalius. Am. J. Neuroradiol., 9:1201-4, 1988.

Martínez, B. F.; Telis, O.; Laza, N. S.; Queirolo, M. F.; Rodríguez, J. \& Grillo, M. Anatomía topográfica del foramen oval con aplicación al tratamiento percutáneo de la neuralgia trigeminal. Rev. Mex. Neuroci., 8(2):1049, 2007.

Reymond, J.; Charuta, A. \& Wysocki, J. The morphology and morphometry of the foramina of the grater wing of the human sphenoid bone. Folia morphol., 64(3):18893, 2005. 
AVILES-SOLIS, J. C.; OLVERA-BARRIOS, A.; DE LA GARZA, C. O.; ELIZONDO-OMAÑA, R. E. \& GUZMÁN-LÓPEZ, S. Prevalencia y características morfométricas del foramen venoso en cráneos del Noreste de México. Int. J. Morphol., 29(I):158-163, 2011.

Sgouros, S.; Natarajan, K.; Hockley, A. D.; Goldin, J. H. \& Wake, M. Skull base growth in childhood. Pediatr. Neurosurg., 31 (5):259-68, 1999.

Sindou, M. \& Tatli, M. Treatment of trigeminal neuralgia with glycerol injection at the gasserian ganglion. Neurochirurgie, 55(2):211-2, 2009.

Sindou, M. \& Tatli, M. Treatment of trigeminal neuralgia with thermorhizotomy. Neurochirurgie, 55(2):203-10, 2009.

Testut, L. Tratado de Anatomía Humana. $7^{\mathrm{a}}$ ed. Barcelona, Salvat Editores, 1924. pp. 156-62. V.1.
Dirección para correspondencia:

J. C. Aviles

Departamento de Anatomía Humana

Facultad de medicina UANL

Av. Madero y Dr Aguirre Pequeño, Sin número

Col. Mitras Centro, Monterrey, NL

MÉXICO

CP 64460

Teléfono: +5281 83294171

Email: j_carlos_aviles@hotmail.com

Recibido : 15-08-2009

Aceptado: 07-12-2010 